\title{
GENERALIZED CONTRACTION MAPPING IN PROBABILISTIC METRIC SPACE
}

\author{
Shams-ur-rahman*, K. Jha \\ Department of Natural Sciences, Kathmandu University, Nepal. \\ *Corresponding address: shams_rahman31@yahoo.com \\ Received 23 February, 2011; Revised 18 April, 2011
}

\begin{abstract}
The probabilistic metric space as one of the important generalization of metric space was introduced by $\mathrm{K}$. Menger in 1942. In this paper, we briefly discuss the historical developments of contraction mappings in probabilistic metric space with some fixed point results.
\end{abstract}

Keywords: Fixed point, Distribution function, t-norm, PM space, contraction mapping.

\section{INTRODUCTION}

Analysis is an important branch of mathematics that was created in the seventeen century during scientific revolution. Since then, no other mathematical field has influenced the development of modern scientific thinking as deeply. Among its several branches, functional analysis appears as a rather complex blend of Algebra and Topology. It does not have only great influence in mathematics but both pure and applied mathematicians are dealing with the nonlinear equations, which have great influence in physical, social, engineering and different technological fields. Analysis has been classified into two major branches: linear and non-linear analysis. Nonlinear analysis as an independent branch of mathematics, has been developed in the 1960's by a great Polish mathematician L. E. J. Browder as a combination of functional analysis and variational analysis and the fixed point theory has unique role for the development of non-linear analysis. The brief historical account of the development of fixed point theorems has been studied by Pant et.al. [14].

The notion of metric space always plays a fundamental role, since continuity in analysis for real and complex functions, depends upon the notion of distance and the generalization of analysis totally depends upon to study the continuity. Metric space was introduced by French mathematician M. Frechet in 1906 and since then there corresponds several generalizations of metric space in the literature. One of important generalization is probabilistic metric space (PM space) which was introduced by Austrian mathematician K. Menger [12] in 1942, using the notion of distribution functions in place of non-negative real numbers. Then, in 1959, A. Sklar and B. Schweizer [16] obtained some fundamental results in probabilistic metric spaces. In 1972, H. Sehgal and A. T. Bharucha-Reid [19] introduced contraction mapping on probabilistic metric space as an extension of famous Banach contraction principle (BCP) to study a fixed point under the special t-norm $\tau_{\mathrm{M}}$. Also, H. Sherwood [21] showed that, for a very large class of 
triangular norms, it is possible to construct complete Menger probabilistic metric space together with the contractive mappings which do not have fixed point. Another type of contraction introduced by T.L. Hicks [8] in 1983, is very convenient definition, which is similar to the classical definition of contraction in metric space. But, in Menger space under minimum t-norm, H. Sehgal and A. T. Bharucha-Reid type contraction is also simple to study several contraction conditions in PM-space, and their usefulness for the establishment of fixed points with applications. Now, the contribution of Menger to resolve the interpretative issue of quantum mechanics has turned out to be of fundamental importance in probabilistic functional analysis and non-linear analysis, e.g.[7]. The purpose of this paper is to study briefly the contraction conditions in probabilistic metric space and its associated properties with some fixed point results.

We have the following definitions.

DEFINITION 1.1: Let $M$ be a set and the distance function $d$ is a mapping from $M \times M$ into $\mathfrak{R}^{+}$, set of non-negative real numbers, satisfying the following conditions:
(d1) $d(x, y) \geq 0$ for $x \neq \mathrm{y}$,
(non-negativity)
(d2) $d(x, y)=0$ iff $x=y$.
( identity of indiscernibles)
(d3) $d(x, y)=d(y, x)$,
(symmetricity),
(d4) $d(x, z) \leq d(x, y)+d(y, z)$,
( subaddivity / triangular inequality),
(d5) $d(x, z) \leq d(x, y) \cup d(y, z)$,
(strong triangular inequality), and
(d6) $d(x, x)=0$,
(reflexivity),

Then, the pair $(M, d)$ is called

1. Metric space if it satisfies conditions (d1), (d2), (d3) and (d4);

2. Pseudometric space if it satisfies conditions (d1), (d3), (d4) and (d6);

3. Quasi-metric space if it satisfies conditions (d1), (d2), and (d4), and

4. Semi- metric space if it satisfies conditions (d1), (d2) and (d3).

DEFINITION 1.2[3]: A triangular norm (shortly t-norm) is a binary operation $T$ (or $\Delta$ or $*)$ on $[0,1]$ which is associative, commutative, non-decreasing at both places and has 1 as the unit element and $T(a, 1)=a$ for every $a \in[0,1]$.

The basic examples are the t-norms $T_{\mathrm{L}}$ (Lukasiewicz t-norm), $T_{\mathrm{P}}$ and $T_{\mathrm{M}}$ (Min norm), defined by $T_{\mathrm{L}}(\mathrm{a}, \mathrm{b})=\max \{\mathrm{a}+\mathrm{b}-1,0\}, T_{\mathrm{P}}(\mathrm{a}, \mathrm{b})=\mathrm{a} \mathrm{b}$ and $T_{\mathrm{M}}(\mathrm{a}, \mathrm{b})=\min \{\mathrm{a}, \mathrm{b}\}$. 
DEFINITION 1.3: A mapping $F: \mathfrak{R} \rightarrow \mathfrak{R}^{+}$is called a distribution function if it is nondecreasing and left-continuous with $\operatorname{Inf}_{\mathrm{t} \in \mathfrak{R}} F(t)=0$, and $\operatorname{Sup}_{\mathrm{t} \in \mathfrak{R}} F(t)=1$.

We denote $\Delta_{+}$to be the class of all distribution functions $F$ such that $F(0)=0$. A distance distribution function is a mapping $\mathrm{F}:[0, \infty) \rightarrow[0,1]$ which is non-decreasing, left continuous on $(0, \infty)$ and $\mathrm{F}(0)=0$. Also, $\mathrm{D}_{+}$is the subset of $\Delta_{+}$containing all functions $\mathrm{F}$ with the condition $\operatorname{Lim}_{t \rightarrow \infty} F(t)=1$.

DEFINITION 1.4: A probabilistic metric space (briefly, a PM-space), is a pair (X, F), where $\mathrm{X}$ is a nonempty set and $F$ is a mapping from $\mathrm{X} \times \mathrm{X}$ to $\Delta_{+}$such that for each $(u, v) \in \mathrm{X} \times \mathrm{X}$, the probabilistic distribution function $F(u, v)$ denoted by $F_{u, v}$ or $F_{\mathrm{uv}}$, are assumed to satisfy the following conditions:

PM1. $\quad F_{u, v}(x)=1$ for every $x>0$ if and only if $u=v$,

PM2. $\quad F_{u, v}(0)=0$ for every $u, v \in \mathrm{X}$,

PM3. $\quad F_{u, v}(x)=F_{v, u}(x)$ for every $u, v \in \mathrm{X}$, and

PM4. If $F_{u, v}(x)=1$ and $F_{u, w}(x)=1$, then $F_{w, v}(x+y)=1$ for every $u, v, w \in \mathrm{X}$.

DEFINITION 1.5: The pair $(\mathrm{X}, F)$ is called a probabilistic semi metric space (shortly PSM space) if (i) $F_{x y}=\varepsilon_{0}$ if and only if $x=y$, and (ii) $F_{x y}=F_{y x}$ for all $x, y \in \mathrm{X}$, where the function $\mathrm{H}=\varepsilon_{0}$, an element of $\mathrm{D}_{+}$, is defined by $\varepsilon_{0}(\mathrm{t})=1$ for $\mathrm{t}>0$ and $\varepsilon_{0}(0)=0$.

DEFINITION 1.6: A probabilistic metric space (or generalized Menger space) is a triple $(X, F, T)$, where $(X, F)$ is PSM space and $T$ is a t-norm with the following condition:

$F_{u, w}(x+y) \geq T\left(F_{u, v}(x), F_{v, w}(y)\right)$ for every $u, v, w \in X$ and $x, y \in \mathfrak{R}^{+}$.

A Menger PM space is a generalized Menger space such that Range $(\mathrm{F}) \subset \mathrm{D}_{+}$.

EXAMPLE 1.1: Let $S=[0$, a $]$ and $F$ be a mapping defined on $S \times S$ with values into $\Delta^{+}$given by $\mathrm{F}_{\mathrm{xy}}=1 / 2\left(\mathrm{H}_{0}+\mathrm{H}_{\mathrm{x}-\mathrm{y}}\right)$ where $\mathrm{H}$ is characteristic function defined on $\mathrm{S} \times \mathrm{S}$ as in definition 1.5. Then, $\left(\mathrm{S}, \mathrm{F}, \tau_{\mathrm{m}}\right)$ is a probabilistic metric space.

DEFINITION 1.7: The order pair (E, T) is called E-space over $(\mathrm{X}, \mathrm{d})$ if $\mathrm{E}$ is the collection of all random variable from a probabilistic space $(\Omega, \mathrm{A}, \mathrm{P})$ into $(\mathrm{X}, \mathrm{d})$ such that for every $\mathrm{p}, \mathrm{q} \in \mathrm{E}$ and every real number $\mathrm{t}$, the set $\{\mathrm{w} \in \Omega: \mathrm{d}(\mathrm{p}(\mathrm{w}), \mathrm{q}(\mathrm{w}))<\mathrm{t}\} \in A$.

DEFINITION 1.8: A topological space is called separable if it contains a countable dense subset, that is, there exists a sequence $\left\{x_{n}\right\}_{n=1}^{\infty}$ of elements of the space such that every nonempty open subset of the space contains at least one element of the sequence. 
DEFINITION 1.9: A topological space $X$ is said to be a Hausdorff space if any two distinct points of $\mathrm{X}$ can be separated by disjoint neighborhoods.

DEFINITION 1.10: If $\mu$ is an extended real valued function defined on a measurable set $\mathrm{E}$, then $\mu$ is a measurable if, for each $\alpha$ in $\mathfrak{R}$, the set $[x: \mu(x)>\alpha]$ is measurable.

DEFINITION 1.11: Let $(X, F, *)$ be a Menger space and * be a continuous t- norm. Then, a sequence $\left\{x_{\mathrm{n}}\right\}$ in a Menger space $(X, F, *)$ is said to converge to a point $x$ in $X$ (written as $x_{n} \rightarrow x$ ) if for every $\varepsilon>0$ and $\lambda \in(0,1)$, there is an integer $\mathrm{n}_{\mathrm{o}}=\mathrm{n}_{\mathrm{o}}(\varepsilon, \lambda)$ such that $F x, x_{n}(\varepsilon)>1-\lambda$ for all $\mathrm{n} \geq \mathrm{n}_{0}$. It is noted that the limit of a convergent sequence in PM space is always unique.

DEFINITION 1.12: The sequence $\left\{x_{\mathrm{n}}\right\}$ is called Cauchy if for every $\varepsilon>0$ and $\lambda \in(0,1)$, there is an integer $\mathrm{n}_{\mathrm{o}}=\mathrm{n}_{\mathrm{o}}(\varepsilon, \lambda)$ such that $F_{x_{n}}, x_{m}(\varepsilon)>1-\lambda$ for all $\mathrm{n}, \mathrm{m} \geq \mathrm{n}_{\mathrm{o}}$. Also, a Menger space $(X, F, *)$ is said to be complete if every Cauchy sequence in it converges to a point of it.

DEFINITION 1.13: A PM space $\mathrm{X}$ is said to be compact if every sequence in $\mathrm{X}$ has a convergent subsequence.

DEFINITION 1.14: Let $f: \mathrm{X} \rightarrow \mathrm{X}$ be a mapping. A point $x$ in $\mathrm{X}$ is a fixed point of $f$ if, $f(x)=x$.

DEFINITION 1.15: A subset $\mathrm{E}$ of $\mathfrak{R}^{\mathrm{n}}$ is a Lebesgue measurable if, for every $\varepsilon>0$, there exist closed set $F$ and open set $U$ such that $F \subset E \subset U$ and $\lambda(U \backslash F)<\varepsilon$. In this case, the Lebesgue measure $\lambda(\mathrm{E})$ of $\mathrm{E}$ is $\lambda^{*}(\mathrm{E})$.

DEFINITION 1.16: Let $(\mathrm{X}, F)$ be a PM space. A self mapping $\mathrm{f}: \mathrm{X} \rightarrow \mathrm{X}$ is called locally power contraction if for each $\mathrm{p} \in \mathrm{X}$, there exists an integer $\mathrm{n}(\mathrm{p})=\mathrm{n}(\mathrm{p}, x)$ where $x$ is positive real number such that $F_{f^{n(p)}(p), f^{n(p)}(q)}(x) \geq F_{p, q}(x / k)$, for some $\mathrm{k}$ in $(0,1)$ and for all $\mathrm{q}$ in $\mathrm{S}$. The powers of $f$ are defined by $f^{0}(\mathrm{p})=\mathrm{p}$, and $f^{\mathrm{n}+1}(\mathrm{p})=f\left(f^{\mathrm{n}}(\mathrm{p})\right), \mathrm{n} \geq 0$.

\section{CONTRACTION CONDITIONS IN PM SPACE}

In this section, two major types of contraction mappings viz B-type contraction and H-type contraction in probabilistic metric space and their recent development in generalized forms, which will be discussed.

We start with the following definition of contraction condition in PM space introduction by H. Sehgal and A. T. Bharucha-Reid in 1972. 
DEFINITION 2.1[19]: Let $(X, F)$ be a PM space. A mapping $\mathrm{T}: X \rightarrow X$ is a contraction mapping (or a SB-Contraction mapping or simply C-contraction) on $(X, F)$ if and only if there exists $\alpha \in(0,1)$ such that $\quad F_{T p, T q}(\mathrm{t}) \geq F_{p, q}(\mathrm{t} / \alpha)$ where $p, q \in X$ and $\mathrm{t}>0$.

DEFINITION 2.2[15]: A self mapping $f$ on a PSM space $(S, F)$ is called a $B$-contraction if there is a $\gamma$ in $(0,1)$ such that for all points $\mathrm{p}, \mathrm{q}$ in $\mathrm{S}$ and all $x>0, F_{f p, f q}(\gamma x) \geq F_{p, q}(x)$.

The following definitions of contraction condition in PM space and PMS space were introduced by T. L. Hicks in 1983.

DEFINITION 2.3[8]: If $(S, F)$ is a PM space, a mapping $f: \mathrm{S} \rightarrow \mathrm{S}$ is called $H$-contraction (also, known as Hicks C-contraction) if there exists $k \in(0,1)$ such that for every $\mathrm{p}, \mathrm{q} \in S$ and $\mathrm{t}>0$, we have $F_{f p, f q}(\mathrm{k} x)>1$ - kt whenever $F_{p, q}(x)>1-\mathrm{t}$.

DEFINITION 2.4[8]: A mapping $f: S \rightarrow S$, where $(S, F)$ is a PSM space, is an $H$-contraction if there is a $k \in(0,1)$ such that for every $p, q \in S$ and every $x>0$, $F_{p, q}(x)>1-x$ implies $F_{f p, f q}(k x)>1-k x$.

Let $(S, F)$ be a probabilistic semi-metric space and for every $p, q \in S$, we have $\beta(\mathrm{p}, \mathrm{q})=\operatorname{Inf}\left\{\mathrm{H}: F_{p, q}\left(\mathrm{~h}^{+}\right)>1-\mathrm{h}\right\}$. If $(\mathrm{S}, F, T)$ is a PM space with $T \geq T_{\mathrm{m}}$, where $\mathrm{t}_{\mathrm{m}}(x, y)=\max \{x+y-1,0\}$, for each $(x, y) \in[0,1]^{2}$, then $\beta$ is a metric on $\mathrm{S}$.

V. Radu, in 1985, showed that every H-contraction in the Menger space $(\mathrm{S}, F, T)$ with $T \geq T_{L}$, where $T_{L}$ is Lukasiewiez t-norm in Menger space, is actually a Banach contraction in the metric space $(\mathrm{S}, \mathbf{K})$, where $\mathrm{K}(p, q)=\sup \left\{\mathrm{t} \geq 0: \mathrm{t} \leq 1-F_{p q}(\mathrm{t})\right\}$ and established the following theorem.

THEOREM 2.1[15]: The mapping $f: \mathrm{S} \rightarrow \mathrm{S}$ is an H-contraction on the PM space $(\mathrm{S}, F, \tau)$ with $\tau \geq \tau_{\mathrm{M}}$ if and only if $f$ is a contraction on the metric space $(\mathrm{S}, \beta)$.

With this condition $\tau \geq \tau_{\mathrm{M}}$, H- contraction on complete PM space has a fixed point, but it is not true for B-contraction. So, it is clear that B-contraction need not be H-contraction. Also, H-contraction need not be B-contraction. For this, we have the following example:

EXAMPLE 2.1[17]: Let $\mathrm{S}=\{0,1,2,3, \ldots\}$ and for $\mathrm{p} \neq \mathrm{q}$, we define $F: \mathrm{S} \times \mathrm{S} \rightarrow \Delta_{+}$such that

$$
F_{\mathrm{pq}}(x)=F_{\mathrm{qp}}(x)=\left\{\begin{array}{lr}
0, & x \leq 2^{-\operatorname{Min}\left(p_{i}, q\right)} \\
1-2^{-\operatorname{Min}\left(p_{i}, q\right)}, & 2^{-\operatorname{Min}\left(p_{i} q\right)}<x \leq 1 . \\
1, & 1<x
\end{array} .\right.
$$


Then, $\left(\mathrm{S}, F, \tau_{\mathrm{M}}\right)$ is a PM space. Also, we define $f: \mathrm{S} \rightarrow \mathrm{S}$ such that $f(\mathrm{r})=\mathrm{r}+1$. Then, $\tau_{\mathrm{M}} \geq \tau_{\mathrm{W}}$ and $\beta(f \mathrm{p}, f \mathrm{q})=\beta(\mathrm{p}, \mathrm{q}) / 2$, which implies that $f$ is an H-contraction. Again, let $\gamma$ be any number in $(0,1)$ and we choose $x \in(1,1 / \gamma)$. Then, we have $\gamma x<1$ and so that

$\mathrm{F}_{f(0) f(1)}(\gamma(x))=\mathrm{F}_{12}(\gamma x) \leq 1 / 2<1=\mathrm{F}_{01}(x)$, whence $f$ is not a B-contraction on $(\mathrm{S}, F)$.

Now, the following lemma explains that B-contraction in a PSM space is stronger than that of $\mathrm{H}$-contraction

LEMMA 2.1[17]: If $f$ is B-contraction on the PSM space (S, $F)$ and if the distribution function $F_{f \mathrm{p}, f \mathrm{q}}$ is strictly increasing on $[0,1]$, then $\beta(f \mathrm{p}, f \mathrm{q})<\beta(\mathrm{p}, \mathrm{q})$.

Proof: We find $\eta$ such that $0<\eta<\frac{1-\gamma}{y} \beta(p, q)$. Then, we have $\beta(p, q)>\gamma[\beta(p, q)+\eta]$.

Since $F_{f p, f q}$ is strictly increasing on $[0,1]$, so $0 \leq \beta(p, q) \leq 1$, and since $f$ is a B-contraction, so we have

$$
\beta(\mathrm{fp}, \mathrm{fq})=F_{\mathrm{fp}, \mathrm{fq}}(\beta(\mathrm{p}, \mathrm{q}))>F_{\mathrm{fp}, \mathrm{fq}}(\gamma[\beta(\mathrm{p}, \mathrm{q})+\mathrm{\eta}]) \geq F_{\mathrm{pq}}(\beta(\mathrm{p}, \mathrm{q})+\mathrm{n}>1-\beta(\mathrm{p}, \mathrm{q}) .
$$

This implies that $\beta(f \mathrm{p}, f \mathrm{q})<\beta(\mathrm{p}, \mathrm{q})$. This completes the proof.

Again, B. Schweizer, H. Sherwood and R.M. Tardiff in 1988 obtained the following relationship between $\mathrm{B}$-contraction and $\mathrm{H}$-contraction in probabilistic semi-metric space.

THEOREM 2.2[17]: Let $(\mathrm{S}, F)$ be a PSM space. Suppose that $\operatorname{Ran}(F)$ is finite and that each element of $\operatorname{Ran}(F) \backslash\left\{\varepsilon_{0}\right\}$ is strictly increasing on $[0,1]$, where $\varepsilon_{0}$ is defined as in definition (1.5). Then, every B-contraction on $(\mathrm{S}, F)$ is an $\mathrm{H}$-contraction.

In general, every B-contraction need not be an H-contraction. To show this, we have the following example:

EXAMPLE 2.2[17]: For each integer $n$, let $p_{n}:(0,1) \rightarrow \mathfrak{R}^{+}$be given by

$\mathrm{p}_{\mathrm{n}}(\mathrm{t})=2^{-\mathrm{n}}(1-\mathrm{t}) \mathrm{t}^{-1}$. Also, let $\mathrm{S}=\left\{\mathrm{p}_{\mathrm{n}}: \mathrm{n}\right.$ is an integer $\}$, let $\mathrm{P}$ be Lebesgue measure on $(0,1)$, and for $x \geq 0$, let $F_{p_{n}, p_{m}}(x)=\mathrm{P}\left\{\mathrm{t} \in(0,1):\left|\mathrm{p}_{\mathrm{n}}(\mathrm{t})-\mathrm{p}_{m}(\mathrm{t})\right|<x\right\}=x /\left(x+\left|2^{-\mathrm{n}}-2^{-\mathrm{m}}\right|\right)$.

Then, a function $f: S \rightarrow \mathrm{S}$ defined by $f\left(\mathrm{p}_{\mathrm{n}}\right)=\mathrm{p}_{\mathrm{n}+1}$, is a B-contraction but is not $\mathrm{H}$ contraction.

LEMMA 2.2: Let $(S, \tilde{F})$ be a PSM space, then $f: S \rightarrow \mathrm{Cl}(\mathrm{S})$, where $\mathrm{Cl}(\mathrm{S})$ is the family of nonempty closed subsets of $\mathrm{S}$, be a B-contraction type mapping such that each element of $\operatorname{Ran}(\tilde{F}) \backslash\left\{\varepsilon_{0}\right\}$ is strictly increasing on $[0,1]$. Then, we have $D_{\beta}(f p, f q) \leq \beta(p, q)$, for every $p, q \in S$.

If $(S, F, \mathrm{t})$ is a Menger space such that $\mathrm{t} \geq \mathrm{t}_{\mathrm{m}}$, then $D_{\beta}(f p, f q)<\beta(p, q)$ for every $p \neq q$. 
THEOREM 2.3[17]: Let $(S, F)$ be a PSM space, and $f: S \rightarrow \operatorname{Com}(\mathrm{S}), \operatorname{Com}(\mathrm{S})$ being nonempty compact subsets of $\mathrm{S}$, be a B-contraction type mapping. If $\operatorname{Ran}(F)$ is finite and each element of Ran $(\widetilde{F}) \backslash\left\{\varepsilon_{0}\right\}$ is strictly increasing on $[0,1]$, then there exists $\gamma \in(0,1)$ such that $D_{\beta}(f p, f q) \leq \gamma \beta(p, q)$, for every $p, q \in S$, where $D_{\beta}$ is defined by

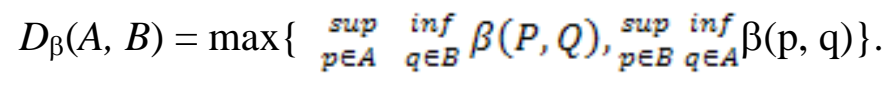

With the above results, we have the following remark.

REMARK: If $(S, F, \mathrm{t})$ is a Menger space such that $\mathrm{t} \geq \mathrm{t}_{\mathrm{m}}$ and that $(S, \beta)$ is a compact metric space, then there exists a fixed point result for f, where f : $S \rightarrow C l(S), C l(\mathrm{~S})$ being the family of nonempty closed subsets of $\mathrm{S}$, with a B-contraction and $\tilde{F}$ is such that every element of $\operatorname{Ran}(F) \backslash\left\{\varepsilon_{0}\right\}$ is strictly increasing.

Now, we discuss some generalized contraction mappings in PM-space with properties.

We consider comparison functions from the class $\Phi$ of all mapping $\varphi:(0,1) \rightarrow(0,1)$ with the properties: (i) $\varphi$ is an increasing bijection, (ii) $\varphi(t)<t$ for all $t \in(0,1)$. It is noted that every such a comparison mapping is continuous. In 1975, Ciric introduced the notion of generalized contraction on a probabilistic metric space and Istratecu [9], in 1981, suggested some interesting problems to study the existence of fixed points of locally power contracting mapping.

DEFINITION 2.5[1]. Let $(X, F)$ be a PM space, a mapping $f: X \rightarrow X$ is said to be a generalized $C$-contraction if there exist a continuous, decreasing function h: $[0,1] \rightarrow$ $[0, \infty]$ such that $\mathrm{h}(1)=0$, and for $m_{1}, m_{2} \in M$, and $k \in(0,1)$ such that the following implication holds: for every $p, q \in X$ and for every $\mathrm{t}>0, \mathrm{ho} F_{\mathrm{p}, \mathrm{q}}\left(\mathrm{m}_{2}(\mathrm{t})\right)<\mathrm{m}_{1}(\mathrm{t})$ implies that $\mathrm{ho} F_{\mathrm{f}(\mathrm{p}), \mathrm{f}(\mathrm{q})}\left(\mathrm{m}_{2}(\mathrm{kt})\right)<\mathrm{m}_{1}(\mathrm{kt})$.

As a generalization of probabilistic B-contraction, we have the following notion of a probabilistic (m, $\mathrm{k}$ )-B-contraction for $\mathrm{m} \geq 1$ and $\mathrm{k} \in(0,1)$.

DEFINITION 2.6 [13]: If $(S, \Phi)$ is a PSM space, $m \geq 1$ and $\mathrm{k} \in(0,1)$, a function $\mathrm{f}: \mathrm{S} \rightarrow \mathrm{S}$ is called probabilistic $(m, k)$-B-contraction if for any $\mathrm{p}, \mathrm{q} \in \mathrm{S}$, there is an $i$ with $1 \leq i \leq \mathrm{m}$ such that for every $\mathrm{t}>0$, we have $\mathrm{F}_{\mathrm{f}}^{\mathrm{i}}$, ${ }_{\mathrm{f}}^{\mathrm{i}}\left(\mathrm{k}^{\mathrm{i}} \mathrm{t}\right) \geq \mathrm{F}_{\mathrm{p}, \mathrm{q}}(\mathrm{t})$.

If $\mathrm{m}=1$ and $\mathrm{k} \in(0,1)$, then a probabilistic $(1, \mathrm{k})$ - $\mathrm{B}$-contraction $\mathrm{f}$ is a probabilistic B-contraction.

DEFINITION 2.7[13]: If $(S, \Phi)$ is a PSM space, $\mathrm{m}>1$ and $\mathrm{k} \in(0,1)$, a function $f: \mathrm{S} \rightarrow \mathrm{S}$ is called probabilistic $(m, k)$-C-contraction if for any $\mathrm{p}, \mathrm{q} \in S$, there is $i$ with $1 \leq i \leq \mathrm{m}$ such that for every $\mathrm{t}>0, \quad F_{\mathrm{p}, \mathrm{q}}(\mathrm{t})>1-\mathrm{t} \Rightarrow F_{\mathrm{fp}}^{\mathrm{i}}, \stackrel{\mathrm{i}}{\mathrm{f}}\left(\mathrm{k}^{\mathrm{i}} \mathrm{t}\right)>1-\mathrm{k}^{\mathrm{i}} \mathrm{t}$.

If $\mathrm{m}=1$ and $\mathrm{k} \in(0,1)$, then a probabilistic $(1, \mathrm{k})$-C-contraction $\mathrm{f}$ is a probabilistic C-contraction. 
If $m_{1}(\mathrm{~s})=m_{2}(\mathrm{~s})=\mathrm{s}$, and $\mathrm{h}(\mathrm{s})=1-\mathrm{s}$ for every $\mathrm{s} \in[0,1]$, we obtain the Hicks definition.

DEFINITION 2.8[17]: A Menger PM-space is a triplet $(E, F, \Delta)$, where $(E, F)$ is a PM space and $\Delta$ is t-norm satisfying the following triangle inequality:

for all $x, y, z \in \mathrm{E}$ and $\mathrm{t}_{1}, \mathrm{t}_{2}>0, \mathrm{~F}_{x, y}\left(\mathrm{t}_{1}, \mathrm{t}_{2}\right) \geq \Delta\left(\mathrm{F}_{x, y}\left(\mathrm{t}_{1}\right), \mathrm{F}_{y}, z\left(\mathrm{t}_{2}\right)\right)$.

In 1960, B. Schweizer, A. Sklar and E. Thorp proved that if $(E, F, \Delta)$ is a Menger $\mathrm{PM}$ space with $\operatorname{Sup}_{0<\mathrm{t}<1} \Delta(\mathrm{t}, \mathrm{t})=1$, then $(E, F, \Delta)$ is a Hausdorff topological space with topology $\mathcal{T}$ induced by the set of $(\varepsilon-\lambda)$-neighborhoods $\left\{\mathrm{U}_{\mathrm{p}}(\varepsilon, \lambda): \mathrm{p} \in \mathrm{E}, \varepsilon>0, \lambda>0\right\}$, where $\mathrm{U}_{\mathrm{p}}(\varepsilon, \lambda)=\left\{x \in \mathrm{E}, \mathrm{F}_{\mathrm{x}, \mathrm{p}}(\varepsilon)>1-\lambda\right\}$. For each two probabilistic bounded subsets $\mathrm{A}$ and $\mathrm{B}$ from $\mathrm{S}$, we have $F_{A, B}(u)=\operatorname{Sup}_{s<u} \operatorname{Inf}_{x \in A} \operatorname{Sup}_{y \in B} F_{x, y}(s)$.

In 1990, O. Hadzic extended $\mathrm{H}$-contraction into the following $\mathrm{H}_{1}$-contraction and $\mathrm{H}_{2}$-contraction type mappings in PSM space.

DEFINITION 2.9[6]: Let $(S, F)$ be a PSM space, $f: S \rightarrow \mathrm{B}(S), \mathrm{B}(S)$ being nonempty bounded subset of $S$ and there exists $\mathrm{k} \in(0,1)$ such that the following implication holds: for every $p, q \in \mathrm{S}$ and $\mathrm{u}>0: F_{p, q}(\mathrm{u})>1-\mathrm{u} \Rightarrow \widetilde{F}_{\mathrm{fp}, \mathrm{fq}}(\mathrm{ku})>1-\mathrm{ku}$. Then, the function $\mathrm{f}$ is an $H_{1}$ - contraction type mapping.

DEFINITION 2.10[5]: Let $(S, F)$ be a PSM space, $f: S \rightarrow \mathrm{n}(S)$ and there exists $\mathrm{k} \in(0,1)$ such that the following implication holds for every $p, q \in \mathrm{S}$ and $x>0$ :

$$
F_{\mathrm{p}, \mathrm{q}}(x)>1-x \Rightarrow \text { for every } u \in f_{p} \text { there exist } \mathrm{v}(u) \in f_{q} \text { such that } F_{\mathrm{u}, \mathrm{v}(u)}(k x)>1-k x \text {. }
$$

Then, the function $f$ is an $\mathrm{H}_{2}$ - contraction type mapping.

THEOREM 2.4[6]: Let (S, F) be a PSM space and $f: \mathrm{S} \rightarrow \mathrm{n}(\mathrm{B}(\mathrm{S}))$ is an $\mathrm{H}_{1}$ - contraction. Then, $f$ is an $\mathrm{H}_{2}-$ contraction and $\mathrm{D}_{\beta}(f \mathrm{p}, f \mathrm{q}) \leq \mathrm{k} . \beta(\mathrm{p}, \mathrm{q})$ for every $\mathrm{p}, \mathrm{q} \in \mathrm{S}$.

REMARK: If $D_{\beta}(f p, f q) \leq \gamma \beta(p, q)$ holds for every $\mathrm{p}, \mathrm{q} \in \mathrm{S}$ and that $F_{\mathrm{p}, \mathrm{q}}(x)>1-x$. Then, this implies that $\beta(\mathrm{p}, \mathrm{q})<x$ and $D_{\beta}(f p, f q) \leq \gamma \beta(p, q)$ implies that $D_{\beta}(f p, f q)<\gamma x$. Using the definition of $\mathrm{D}_{\beta}$, we have $\underset{\text { uefp }}{\sup } \underset{v \in f q}{\inf } \beta(u, v)<\gamma x$ and $\underset{v \in f p}{\sup } \underset{\text { unf } f q}{\sin } \beta(u, v)<\gamma x$. Then, we have $\inf _{v \in f q} \beta(u, v)<\gamma x$, for every $u \in f p$ and $\underset{u \in f q}{\inf } \beta(u, v)<\gamma x$, for every $v \in f q$. Since $f p$ and $f q$ are compact for every $u \in f p$, there exists $v(u) \in f q$ such that $F_{u, v}(u)(\gamma x)>1-\gamma x$. Thus, $f$ is $\mathrm{H}_{2-}$ contraction.

LEMMA 2.4[3]: Let $(S, F, \Delta)$ be a generalized Menger space, $\Delta$ be a t- norm satisfying $\lim _{t \rightarrow 1-} \Delta(t, s)=s$ for all $\mathrm{t}, \mathrm{s} \in[0,1]$ and let $\left\{p_{n}\right\},\left\{q_{n}\right\}$ be two sequences in $\mathrm{S}$ with 
$\mathrm{p}_{\mathrm{n}} \stackrel{T}{\rightarrow} \mathrm{p}, \mathrm{q}_{\mathrm{n}} \stackrel{T}{\rightarrow} \mathrm{q}$, respectively. Then, we have

(1) For any given $\mathrm{t} \in \mathrm{R}$, $\liminf _{n \rightarrow \infty} F p_{n_{s}} q_{n}(\mathrm{t}) \geq F_{p, q}(\mathrm{t})$.

(2) If $\mathrm{t} \in \mathfrak{R}$ is a continuous point of $F_{p, q}$, then $\lim _{n \rightarrow \infty} F p_{n_{2}} q_{n}(\mathrm{t})=F_{p, q}(\mathrm{t})$.

In 1996, S.S. Chang, S.S. Lee, Y.J. Cho, Q.Y. Chen, S.M. Kang and J.S. Jung defined the following Menger probabilistic normed space.

DEFINITION 2.11[3]: A triplet $(E, F, \Delta)$ is called a Menger probabilistic normed space (briefly, a Menger PN-space) if $E$ is a real vector space, $F$ is a distribution function from $E$ into $\mathrm{D}$ and $\Delta$ is a $t$-norm satisfying the following conditions:

PN1. $F_{x}(0)=0$,

PN2. $F_{x}(\mathrm{t})=\mathrm{H}(\mathrm{t})$ for all $\mathrm{t}>0$ if and only if $\mathrm{x}=0$,

PN3. $F_{\alpha x}(\mathrm{t})=F_{\alpha x}(\mathrm{t} /|\alpha|)$ for all $\alpha \in \mathrm{R}, \alpha \neq 0$, and

PN4. $F_{x+y}\left(\mathrm{t}_{1}+\mathrm{t}_{2}\right) \geq\left(F_{x}\left(\mathrm{t}_{1}\right), F_{y}\left(\mathrm{t}_{2}\right)\right)$ for all $x, y \in \mathrm{E}$ and $\mathrm{t}_{1}, \mathrm{t}_{2} \in \mathfrak{R}^{+}$.

DEFINITION 2.12: Let $(E, F, \Delta)$ be a Menger probabilistic space. Then, a subset of $E$ is said to be probabilistically bounded if $\begin{gathered}\text { sup inf } \\ t>0 \\ x y y \in A\end{gathered} F_{x y y}(\mathrm{t})=1$.

In 2005, D. Mihet introduced the following weak-Hicks contraction in probabilistic semimetric space as a generalization of Hicks contraction.

DEFINITION 2.13[13] Let $(S, F)$ be a probabilistic semimetric space. A self mapping A of $\mathrm{S}$ is called a weak-Hicks contraction (shortly, wH contraction) if there exists $\mathrm{k} \in(0,1)$ such that for all $\mathrm{p}, \mathrm{q} \in \mathrm{S}$ and each $\mathrm{t} \in(0,1)$, we have

$F_{p q}(\mathrm{t})>1-\mathrm{t}$ implies $F_{A(p), A(q)}(\mathrm{kt})>1-\mathrm{kt}$.

The class of wH-contraction is strictly larger than that of C-contraction. It is noted that every $\mathrm{wH}$-contraction is (uniformly) continuous [13].

DEFINITION 2.14 [13]: A PSM space $(\mathrm{S}, F)$ is an $H$-space if it satisfies the triangle inequality: for $\varepsilon>0$, there exists $\delta>0, F_{\mathrm{pq}}(\delta)>1-\delta, F_{\mathrm{qr}}(\delta)>1-\delta$, this implies $F_{\text {pr }}(\varepsilon)>1-\varepsilon$.

It is noted that every Menger space $(\mathrm{S}, F, T)$ with $T$ satisfying $\operatorname{Sup}_{a<1} T(a, a)=1$, is an H-space. In 2009, A. Beitollahi and P. Azhdari introduced the following multi-valued mapping $(\varphi-\mathrm{k})-\mathrm{B}$ contraction, as a generalization of B-contraction for single valued mapping introduced by D. Mihet [13].

DEFINITION 2.15[2]: Let $S$ be a nonempty set, $\varphi \in \Phi, k \in(0,1)$, and $\mathrm{F}$ be a probabilistic distance on $\mathrm{S}$. Then, a mapping $\mathrm{f}: \mathrm{S} \rightarrow 2^{\mathrm{S}}$ is called a multi-valued $(\varphi-k)-$ $B$ contraction if for every $x, y \in \mathrm{S}, \varepsilon>0$ and for all $\mathrm{t} \in(0,1)$ the following implication holds: $\quad F_{x y}(\varepsilon)>1-\mathrm{t} \Rightarrow$ for each $\mathrm{p} \in f_{x}$ there corresponds $\mathrm{q} \in f_{\mathrm{y}}: F_{p q}(\mathrm{k} \varepsilon)>1-\varphi(\mathrm{t})$, 
where $2^{\mathrm{S}}$ denotes the class of all nonempty subsets of the set $\mathrm{S}$ and $\mathrm{C}(\mathrm{S})$ is the class of all nonempty closed (in the F-topology) subsets of $\mathrm{S}$.

DEFINITION 2.16[12]: Let $(\mathrm{X}, F)$ be a PSM space and $\varepsilon \in(0,1)$. A mapping $f: \mathrm{X} \rightarrow \mathrm{X}$ is called probabilistic $\varepsilon$-contractive if $F_{f(x), f(y)}(t)>F_{x y}(t)$ whenever $F_{x y}(\mathrm{t})>1-\varepsilon$ and $x \neq y$.

\section{SOME FIXED POINT RESULTS IN PM SPACE}

In this section, we state some fixed point results for single self maps in PM and PSM spaces. In 1981, V.I. Istratecu proposed the interesting problem of studying the existence of fixed points of locally power contracting mapping.

THEOREM 3.1: A sequence $\left\{p_{\mathrm{n}}\right\}$ in a PM space $X$ converges to $\mathrm{p}$ if and only if for every $x \in \mathfrak{R}$, we have $\lim F_{p, p n}(x)=\mathrm{H}(x)$.

THEOREM 3.2[1]: Let $(X, F)$ be a complete PM space, and if for every $p \in X$, there exists a positive integer $n(p)=n(p, x)$ such that $\mathrm{F}_{\mathrm{f}} n_{(\mathrm{p}), \mathrm{f}} \mathrm{n}_{(\mathrm{q})}(x) \geq \varphi\left(D_{\mathrm{Op}, \mathrm{q}(\mathrm{f})} \mathrm{O}(x)\right)$, where $\mathrm{n} \geq \mathrm{n}(\mathrm{p})$ and $\mathrm{q} \in X$. Then, there exists $\mathrm{p} \in X$ such that $\mathrm{F}_{\mathrm{f}} \mathrm{k}_{(\mathrm{q})}, \mathrm{p}(x) \rightarrow \mathrm{H}(x)$ as $\mathrm{k} \rightarrow \infty$ for $\mathrm{q} \in X$.

In 1990, O. Hadzic introduced the notion of a multi-valued probabilistic $\varphi$-contraction by using the notion of non-compactness, which is as follows:

THEOREM 3.3[6]: Let $(\mathrm{S}, F)$ be a PSM space and $\mathrm{f}: \mathrm{S} \rightarrow \operatorname{Com}(\mathrm{S}), \operatorname{Com}(\mathrm{S})$ being a nonempty compact subsets of $\mathrm{S}$, be a B-contraction type mapping. If $\operatorname{Ran}(F)$ is finite and each element of $\operatorname{Ran}(F) \backslash\left\{\varepsilon_{0}\right\}$ is strictly increasing on $[0,1]$, then there exists $\gamma \in(0,1)$ such that $D_{\beta}(f p, f q) \leq \gamma \beta(p, q)$, for every $p, q \in S$, where $D_{\beta}$ is defined by:

$D_{\beta}(A, B)=\max \left\{\sup _{p \in A} \ln f_{q \in B} \beta(p, q), \sup _{p \in B} \inf f_{q \in A} \beta(p, q)\right\}$.

In 1992, I. Beg, S. Rahman and N. Shahzad proved following fixed point theorem in complete PM space.

THEOREM 3.4[1]: Let $(X, F)$ be a complete PM space. If for every $\mathrm{p} \in \mathrm{X}$, there exists a positive integer $\mathrm{n}(\mathrm{p})=\mathrm{n}(\mathrm{p}, x)$ such that $F_{f(\mathrm{p}), \mathrm{f}(\mathrm{q})}^{\mathrm{n}}(x) \geq \phi\left(D_{\mathrm{Op}, \mathrm{q}(\mathrm{f})} \mathrm{O}(x)\right)$, where $\mathrm{n} \geq \mathrm{n}(\mathrm{p})$ and $\mathrm{q} \in X$. Then, there exists $\mathrm{p}$ in $\mathrm{X}$ such that $F_{f} k_{(q), p}(x) \rightarrow H(x)$ as $k \rightarrow \infty$ for $\mathrm{q}$ in $X$.

THEOREM 3.5[1]: Let $(X, F, \tau)$ be a complete PM space under $\tau$ with

$\sup \{\tau(F, F): F<H\}$. If for every $\mathrm{p} \in X, F_{f(\mathrm{p}) \mathrm{f}(\mathrm{q})}(x) \geq \varphi\left(D_{\mathrm{Op}, \mathrm{q}(\mathrm{f})} \mathrm{O}(x)\right)$, where $\mathrm{q} \in X$, then $\mathrm{f}$ has a fixed point and there exists a point $\mathrm{p}$ in $X$ such that $F_{f} k_{(q), p}(x) \rightarrow H(x)$ as $k \rightarrow \infty$ for any q in $X$.

In 1996, S. S. Chang, B. S. Lee, Y. J. Cho, Y. Q. Chen, S. M. Kang, and J. S. Jung proved the following fixed point theorems in complete PN space.

THEOREM 3.6[3]: Let $(E, F, \Delta)$ be a complete PM space with a t-norm $\Delta$ satisfying $\Delta(\mathrm{t}$, $\mathrm{t}) \geq \mathrm{t}$ for all $\mathrm{t} \in[0,1]$. Let $T: E \rightarrow E$ be a mapping with 


$$
F_{\mathrm{T}_{x}, \mathrm{~T}_{y}}(\mathrm{t}) \geq F_{x, y}(\mathrm{t} / \mathrm{k}(\alpha, \beta))
$$

for all $x, y \in E, \mathrm{t} \geq 0$ and $\alpha, \beta \in(0,+\infty)$ with $F_{x y}(\alpha)>0$ and $F_{x, y}<1$, where $\mathrm{k}(\alpha, \beta)$ : $(0,+\infty)^{2} \rightarrow(0,1)$ is a function. Then, $\mathrm{T}$ has exactly one fixed point in $E$.

In the sequel, the mapping $\mathrm{T}$ that satisfies (3.1) is called a generalized contraction mapping on E.

THEOREM 3.7[3]: Let $(E, F, \Delta)$ be a complete Menger PN space, $C$ be a probabilistically bounded closed convex subset of $E, T: C \rightarrow E$ be a generalized contraction mapping defined on $C$ and $S: C \rightarrow E$ be a continuous mapping with $\mathrm{S}(\mathrm{C})$ being relatively compact. If $T_{x}+T_{y} \in C$ for all $x, y \in C$, then $T+S$ has a fixed point in C.

In 2006, M.B. Ghaemi and A. Razani established fixed point and periodic point for single self mappings in Menger PM space.

THEOREM 3.8.[5]: Let (S, F, T) be a Menger PM space. If $\mathrm{A}$ is a C-contraction on $\mathrm{S}$ and for some $\mathrm{u}$ in $\mathrm{S}$, then $\left(A^{n_{i}}(u)\right)$ is a convergent subsequence of $\left(\mathrm{A}^{\mathrm{n}}(\mathrm{u})\right)$, and the point $\xi=\operatorname{Lim}_{n \rightarrow \infty} A^{n_{i}}(u)$ is the unique fixed point of $\mathrm{A}$.

In 2008, D. Mihet proved following fixed point results for weak Hick's contraction.

THEOREM 3.9[13]: Let $(X, F)$ be an $\mathrm{H}$ space and A be a weak - Hicks contraction on $X$. If there exists $x \in X$ such that $F_{x A(x)}(1)>0$ and the sequence $\left(\mathrm{A}^{\mathrm{n}}(\mathrm{p})\right)_{\mathrm{n} \in \mathrm{N}}$ has a convergent subsequence, then $\left(\mathrm{A}^{\mathrm{n}}(\mathrm{p})\right)$ converges to a fixed point of $\mathrm{A}$.

EXAMPLE 3.1: Let $F_{x y}(t)=\frac{\min (x, y)}{\max (x, y)}$, for all $\mathrm{t} \in(0, \infty)$, and for all $x, y \geq 0, x \neq y$. Then, $\mathrm{X}$ $=[0, \infty)$ is a complete generalized Menger space under the triangular norm $T_{P} \geq T_{L}$. Also, the mapping $\mathrm{g}: \mathrm{X} \rightarrow \mathrm{X}$ defined by $\mathrm{g}(x)=1$, if $x>0$, and $\mathrm{g}(0)=0$, is a wH-contraction. This mapping has two fixed points: $x=0$ and $x=1$. So, unlike C-contractions, a weak H-contraction is not necessarily a Banach contraction on the metric space $(\mathrm{S}, \mathbf{K})$, where

$\mathbf{K}(p, q)=\sup \left\{\mathrm{t} \geq 0: \mathrm{t} \leq 1-F_{p q}(\mathrm{t})\right\}$.

THEOREM 3.10[11]: Let $(\mathrm{X}, F, T)$ be a Menger PM space with the t-norm $T$ satisfying the condition of Lemma 2.4 and $f: \mathrm{X} \rightarrow \mathrm{X}$ be a probabilistic $\varepsilon$-contractive mapping. Suppose that for some $x \in \mathrm{X}$, the sequence $\left(f^{\mathrm{n}}(x)\right), \mathrm{n} \in \aleph$, contains a convergent subsequence with $x^{*}$ as its limit. Then, $x^{*}$ is the unique fixed point of $f$.

In 2009, A. Beitollahi and P. Azhdari proved the following fixed point theorems for multi-valued contraction on complete Menger space.

THEOREM 3.11[2]: Let $(\mathrm{S}, F, T)$ be a complete Menger space with $\sup _{0 \leq \mathrm{a}<1} T(\mathrm{a}, \mathrm{a})=1$ and $f: \mathrm{S} \rightarrow \mathrm{C}(\mathrm{S})$ be a multi-valued $(\varphi-\mathrm{k})-\mathrm{B}$ contraction. If there exist $\mathrm{p} \in \mathrm{S}$ and $\mathrm{q} \in f \mathrm{p}$ 
such that $F_{\mathrm{pq}} \in \mathrm{D}_{+}$and $\lim _{\mathrm{n} \rightarrow \infty} T_{\mathrm{i}=1}^{\infty}\left(1-\varphi^{\mathrm{n}+\mathrm{i}-1}(\lambda)\right)=1$, for every $\lambda \in(0,1)$, then $f$ has a fixed point.

THEOREM 3.12[2]: Let $(\mathrm{S}, F, T)$ be a complete Menger space, $T$ a t-norm such that $\sup _{0 \leq \mathrm{a}<1} T(\mathrm{a}, \mathrm{a})=1, \mathrm{M}$ a nonempty and closed subset of $\mathrm{S}, f: \mathrm{M} \rightarrow \mathrm{C}(\mathrm{M})$ be a multivalued $(\varphi-\mathrm{k})-\mathrm{B}$ contraction and also weakly demicompact. If there exist $x_{0} \in \mathrm{M}$ and $x_{1} \in f x_{0}$ such that $F_{x 0 x 1} \in \mathrm{D}_{+}$, where $\mathrm{D}_{+}$being the set of all distribution functions, then $\mathrm{f}$ has a fixed point.

\section{ACKNOWLEDGEMENT}

The authors would like to thank Prof. Dr. Ismat Beg from Lahore University of Management Science (LUMS), Pakistan and Dr. Javed Ali from Aligarh Muslim University (AMU), India for regular kind cooperation sending their research papers.

\section{REFERENCES}

[1] Beg I, Rahman S \& Shazad N, Fixed points of generalized contraction mappings on probabilistic metric spaces, Pak. J. Stat., 2(8) (1992), 35.

[2] Beitollahi A \& Azhdari P, Multi-valued contraction theorems in probabilistic metric spaces, Internat. J. Math. Anal., 3(24) (2009), 1169.

[3] Chang S S, Lee B S, Cho Y J , Chen Y Q, Kang S M \& Jung J S, Generalized contraction mapping and differential equation in probabilistic metric spaces, Proc. Amer. Math. Soc., 124(8) (1996), 2367.

[4] Caristi J , Fixed point theorems for mapping satisfying inwardness conditions, Trans. Amer. Math. Soc., 215(1976), 241.

[5] Ghaemi M B \& Razani A, Fixed and periodic points in a probabilistic normed and metric spaces, Chaos, Solitons \& Fractals, 28(2006), 902.

[6] Hadzic O, On multivalued contractions in probabilistic metric spaces, Univ. u Novom Sadu Zb. Rad. Prirod.-Mat. Fak. Ser. Mat., 2 (20) (1990), 161.

[7] Hadzic O \& Pap E, Fixed point theory in probabilistic metric spaces, Kluwer Academic Publ., Dordrecht, 2001

[8] Hicks T L, Fixed point theory in probabilistic metric spaces, Rev. Rech. Novi. Sad, 13 (1983), 63. 
[9] Istratescu V I, Fixed point theory: an introduction, D. Reidel, Holland, 1981, ISBN No. 90-227-1224-7.

[10] Kutuckcu S, A fixed point theorem for contraction type mapping in Menger spaces, Amer. J. Appl. Sci., 6(4)(2007), 371.

[11] Lafuerza-Guillen B \& Rafi M, Generalized Banach contraction in probabilistic metric/normed spaces, arxiv:0706.1418vl. [math.FA] 11 Jun 2007.

[12] Menger K, Statistical metrics, Proc. Nat. Acad. Sci., 28 (1942), 535.

[13] Mihet D, Fixed point theorems in probabilistic metric spaces, Chaos, Solitons, Fractals, 41(2008), 1014.

[14] Pant R P, Lohani A B \& Jha K, A history of fixed point theorems, GANITA BHARATI: Bull. Soc. Hist. Math. India, 24(1-4)(2004), 147.

[15] Radu V, On some fixed point theorems in probabilistic metric spaces, Lecture Notes in Mathematics, 1233 (1985), 125.

[16] Schweizer B \& Skalar A, Statistical metric spaces, Pacific J. Math., 10(1959), 313.

[17] Schweizer B, Sherwood H \& Tardiff R M, Contractions on probabilistic metric spaces: Examples and counterexamples, Stochastica XII-1 (1988), 5.

[18] Schweizer B, Skalar A \& Thorp E, The metrization of statistical metric spaces, Pacific J. Math., 10 (1960), 673.

[19] Sehgal H \& Bharucha-Reid A T, Fixed points of contraction mappings on probabilistic metric spaces, Math. System Theory, 6 (1972), 97.

[20] Shams-ur-Rahman \& Jha K, A study on statistical metric space in analysis, In Proceeding of National Conference of Mathematics (NCM 2010): a publication of Nepal Mathematical Society, p.p. 60, 17- 19 January, 2010.

[21] Sherwood H, Complete probabilistic metric spaces, Z. Wahr, Verw. Gab., 20(1971), 117. 